\section{Adjuvantes Trastuzumab: Nutzen versus kardiales Risiko}

Trastuzumab in Verbindung mit Chemotherapie hat sich als effektiv in der adjuvanten Behandlung des HER2-positiven Mammakarzinoms erwiesen. Vor allem für Anthrazyklin-haltige Regimes ist eine erhöhte Kardiotoxizität bekannt. Jetzt liegt eine Nutzen-Risiko-Analyse dieses Ansatzes im Hinblick auf die kardiale Sicherheit nach 7-Jahres-Follow-up vor.

m Rahmen der Phase-III-Studie B-31 des National Surgical Adjuvant Breast and Bowel Project (NSABP B-31) kamen 1.830 Frauen mit einem nodal positiven, HER2-positiven Mammakarzinom bezüglich kardialer Ereignisse zur Auswertung. Die Teilnehmerinnen waren in zwei Therapiearme randomisiert.

1. ACP: vier Zyklen Doxorubicin $(60 \mathrm{mg} /$ $\mathrm{m}^{2}$ ) und Cyclophosphamid (600 mg/ $\mathrm{m}^{2}$ ) gefolgt von entweder vier Zyklen Paclitaxel $\left(175 \mathrm{mg} / \mathrm{m}^{2}\right)$ in dreiwöchigen Intervallen oder zwölf Wochendosen Paclitaxel $\left(80 \mathrm{mg} / \mathrm{m}^{2}\right)$.

2.ACPH: ACP plus wöchentlich Trastuzumab (4 mg/kg initial, dann $2 \mathrm{mg} /$ $\mathrm{kg}$ ) über 52 Wochen, beginnend mit der ersten Paclitaxel-Dosis.
Die Studie lief von 2000 bis 2005; die Autoren präsentieren nun ein 7-JahresUpdate zur kardialen Sicherheit. Berücksichtigt wurde auch die Erholung von einem kardialen Vorfall anhand der linksventrikulären Auswurffraktion (LVEF). Die Autoren entwickelten ein statistisches Modell zur Risikoabschätzung einer schweren Herzinsuffizienz.

In dem Beobachtungszeitraum erlitten $4 \%$ der Patientinnen aus dem Trastuzumab-Arm kardiale Nebenwirkungen gegenüber $1,3 \%$ aus der ACP-Gruppe. In beiden Armen kam es zu einem kardial bedingten Todesfall. Der kardiale Risikoscore der Autoren berücksichtigte das Alter und die LVEF vor Be- handlungsbeginn. Demnach könnten Patientinnen mit einem höheren Wert von Regimes ohne Anthrazykline profitieren, während ein niedriger Score für die ACPH-Behandlung prädestiniert, so die Autoren. Bei der Mehrheit der Patientinnen mit kardialen Nebenwirkungen normalisierte sich die LVEF nach Absetzen von Trastuzumab. Nur zwei kardiale Spätfolgen traten mehr als zwei Jahre nach Start der Trastuzumab-Behandlung auf.

Fazit: Nach der aktuellen Datenlage liegt das Nutzen-Risiko-Verhältnis einer adjuvanten Trastuzumab-Therapie gemäß NSABP-Protokoll 31 eindeutig auf Seite der Behandlung mit dem monoklonalen Antikörper. Herzinsuffizienz als Spätfolge dieses Regimes ist sehr ungewöhnlich, so die Autoren. Wolfgang Zimmermann

Romond EH et al. Seven-year follow-up assessment of cardiac function in NSABP B-31, a randomized trial comparing doxorubicin and cyclophosphamide followed by paclitaxel (ACP) with ACP plus trastuzumab as adjuvant therapy for patients with node-positive, human epidermal growth factor receptor 2-positive breast cancer. J Clin Oncol. 2012;30(31):3792-9.

\title{
Erneutes Ansprechen dank Konjugat-Präparat T-DM1 bei metastasiertem HER2-positivem Brustkrebs
}

Für mehrfach vorbehandelte Patientinnen mit metastasiertem HER2positiven Mammakarzinom sind die Therapieoptionen beschränkt. In dieser Situation könnte das Antikörper-Zytostatikum-Konjugat T-DM1 zu einem erneuten Ansprechen und verlängerten progressionsfreien Intervall führen.

$\mathrm{D}$ iese These wurde in einer Phase-IIStudie bei 110 Brustkrebspatientinnen mit nachgewiesener HER2-Überexpression überprüft. Die Vorbehandlung umfasste multiple Chemotherapien mit einem Anthrazyklin, einem Taxan und Capecitabin und gegen HER2 gerichtete Regimes (Trastuzumab, Lapatinib).

Trastuzumab bindet an die extrazelluläre HER2-Domäne und kann als Kombinationspartner einer Chemotherapie bei HER2-Positivität das Gesamtüberleben beim metastasierten Mammakarzinom (MBC) verlängern. Der HER1-/ HER2-Tyrosinkinase-Inhibitor Lapatinib verlängert in Verbindung mit Capecitabin das progressionsfreie Überleben.
Das Antikörper-Wirkstoff-Konjugat Trastuzumab-Emtansin (T-DM1) kombiniert die intrazelluläre Wirkung von Emtansin gegen Mikrotubuli mit der Anti-Tumor-Aktivität des monoklonalen Antikörpers.

Primäre Studienziele waren Gesamtansprechen und Therapiesicherheit, sekundäres Ziel die klinische Benefitrate. Die Patientinnen erhielten $3,6 \mathrm{mg} / \mathrm{kg}$ des Konjugats in dreiwöchigen Zyklen. Die Gesamtansprechrate betrug 34,5\% und die klinische Benefitrate $48,2 \%$. Das mediane progressionsfreie Intervall betrug 6,9 Monate, die mediane Ansprechdauer 7,2 Monate. Patientinnen mit retrospektiv bestätigter HER2-Positivität sprachen mit $41,3 \%$ besser an. Die meisten unerwünschten Ereignisse waren vom Grad 1/2. Dabei dominierten Thrombozytopenien mit 9,1\%.

Fazit: Das Antikörper-Wirkstoff-Konjugat Trastuzumab-Emtansin (T-DM1) ist gut verträglich und aktiv bei Patientinnen mit metastasiertem, HER2-positiven Mammakarzinom, die extensiv mit HER2-gerichteten Agenzien und multiplen Chemotherapeutika vorbehandelt sind. Für diese Patientinnen könnte sich T-DM1 als effektive neue Behandlungsstrategie etablieren. Wolfgang Zimmermann

Krop IE et al. A phase II study of trastuzumab emtansine in patients with human epidermal growth factor receptor 2-positive metastatic breast cancer who were previously treated with trastuzumab, lapatinib, an anthracycline, a taxane, and capecitabine. J Clin Oncol. 2012;30(26): 3234-41. 